\title{
Acanthosis nigricans in adolescents with polycystic ovary syndrome
}

\author{
Bijay Khan*, Ranjan Basu
}

Department of Obstetrics and Gynecology, KPC Medical College, Kolkata, West Bengal, India

Received: 01 February 2022

Revised: 16 February 2022

Accepted: 17 February 2022

\author{
*Correspondence: \\ Dr. Bijay Khan, \\ E-mail: drbkhan2015@gmail.com
}

Copyright: (c) the author(s), publisher and licensee Medip Academy. This is an open-access article distributed under the terms of the Creative Commons Attribution Non-Commercial License, which permits unrestricted non-commercial use, distribution, and reproduction in any medium, provided the original work is properly cited.

\begin{abstract}
Background: In this study we compared the clinical and biochemical variables of adolescent females with PCOS (polycystic ovarian syndrome) and its association with or without acanthosis nigricans (AN).

Methods: Adolescent girls (14-19 years) with oligomenorrhoea and hyperandrogenism were studied. Clinical parameters like blood pressure (BP), body mass index (BMI), abdominal circumference (AC), presence or absence of acne and AN and hirsutism score were noted. Biochemical tests included serum total testosterone (TT), sex hormone binding globulin (SHBG) levels and free androgen index (FAI). Postprandial plasma insulin (PPI) and glucose (PPG) levels were measured to assess insulin resistance and glucose tolerance respectively.

Results: Significant differences were observed in BMI, AC, DBP, TT and FAI between the two groups. The difference in the prevalence rate of abnormal glucose tolerance and insulin resistance between the two groups was insignificant. Logistic regression modelling with AN as the response variable of interest and BMI, AC, SBP, DBP, testosterone level, PP insulin and PPG levels as its predictors yields BMI, testosterone, PP sugar, PPI, SHBG as main determinants. The model signifies positive impacts of BMI and testosterone level, while negative influence of PP sugar, PPI level and SHBG levels on AN. This analysis was an evidence for a strong relation between BMI and AN.

Conclusions: AN in adolescent girl with PCOS is another clinical marker of obesity but not an indicator of underlying insulin resistance or glucose intolerance. Further studies are needed to detect how many of them ultimately develop insulin resistance or diabetes in future.
\end{abstract}

Keywords: Acanthosis nigricans, Adolescent, Body mass index, Modified Ferriman-Gallwey, Polycystic ovary syndrome, Risk factors

\section{INTRODUCTION}

AN is an easily visible, cosmetic paraneoplastic dermatologic condition characterized by brownish-black, velvety thickening, papillomatous, hyperkeratotic plaques. It is typically observed on the intertriginous areas of the skin, commonly in pigmented population. ${ }^{1,2} \mathrm{AN}$ was first reported in the International atlas for rare skin diseases about 130 years ago and the term was proposed by Paul Gerson Unna, Hamberg, Germany. ${ }^{3} \mathrm{AN}$ is a manifestation of several underlying metabolic defect like insulin resistance (IR), obesity, PCOS and hyperandrogenism
(HA). ${ }^{3-6}$ Studies show that between 5 to $33 \%$ of patients with PCOS have AN. ${ }^{6,7} \mathrm{AN}$ is also rarely associated with other pathological conditions like insulinoma and malignant diseases, especially adenocarcinoma of the stomach. ${ }^{8}$

AN is more common in obese PCOS patients. ${ }^{9}$ The histological characteristics of AN are mainly hyperkeratosis and papillomatosis changes of the skin. As early as in 1976, Kahn et al reported an association of HA and insulin resistance with AN. ${ }^{10}$ Since then $\mathrm{AN}$ is often detected in HA and diabetes mellitus patients. ${ }^{11-13}$ 
There are scanty studies which have reported the clinical, hormonal and metabolic parameters in PCOS with AN. In the present paper we studied various clinical and biochemical variables (including hormonal and metabolic parameters) of adolescent girls having PCOS with and without AN.

\section{METHODS}

The present comparative cross-sectional observational research was conducted at the KPC medical college, Kolkata, India, which was a tertiary care hospital, for a duration of 24 months (April 2017 to March 2019). Clearance was obtained from the human ethical committee of the institute and all females candidates were duly informed about the investigation procedure.

The data were collected at the time of encounter from 59 adolescence girls between the age of 14-19 years, with PCOS. A complete demographic data including their age, socio-economic status, habitat along with family history of $\mathrm{DM}$ in first degree relative was noted. As per the Rotterdam 2003 criteria, these girls were subjected to detailed clinical and biochemical evaluations for the diagnosis of PCOS, with at least two of the following characteristics: chronic anovulation or oligo-ovulation; hyperandrogenism (clinical and/or biochemical) and (polycystic ovaries appearance under transabdominal ultrasound. ${ }^{14}$ As per the Rotterdam criteria, secondary causes of hyperandrogenism were excluded by relevant clinical and biochemical laboratory tests. Women with reported history of previously diagnosed diabetes, steroid or oral contraceptives consumption in the prior to 3 months of the study were also eliminated from the study.

The feature oligo-ovulation and/or anovulation was indicated by oligomenorrhea (intermenstrual intervals of $\geq 35$ days) and amenorrhea (intervals $>3$ months). The presence of acne, hirsutism (modified Ferriman-Gallwey mFG score of $\geq 6$ ) or androgenic alopecia were taken as clinical criterion of hyperandrogenism. Total testosterone level more than $0.82 \mathrm{ng} / \mathrm{ml}$ (normal laboratory range 0.06 $0.82 \mathrm{ng} / \mathrm{ml}$ ) or calculated free androgen index more than 2.06 was considered as biochemical hyperandrogenism. ${ }^{14}$ The detection of not less than one ovary $10 \mathrm{cc}$ or more in volume during transabdominal ultrasound was considered as polycystic ovary.

A questionnaire-based survey was conducted to document the personal and family history of diabetes, obesity, hypertension and/or ischemic heart disease along with the length of menstrual cycles. Symptoms of hyperandrogenism (hirsutism, persistent acne and/or oily skin, and androgenic alopecia), existence of acanthosis and insulin resistance were noted during the physical examination. The anthropometric measurements encompassed abdominal and hip circumference $(\mathrm{cm})$ using measuring tape. BMI $\left(\mathrm{kg} / \mathrm{m}^{2}\right)$ was calculated from height $( \pm 0.5 \mathrm{~cm})$ and weight $( \pm 0.5 \mathrm{~kg})$ measurements. Waist and hip ratio (WHR) was measured using measuring tape. Weight was measured on a digital platform weighing scale. Both SBP and DBP were measured using a mercury sphygmomanometer $(\mathrm{mm}$ of $\mathrm{Hg})$ as per standard procedure.

Fasting plasma glucose (FG) (expressed in $\mathrm{mg} \%$ ) and FI levels (in $\mathrm{mcu} / \mathrm{ml}$ ) were measured after overnight fasting ( 12 hour) for all candidates. Glucose oxidase peroxidase method (Roche Diagnostics Gmbh, Mannheim, Germany) was used to measure the plasma glucose. Glucose-insulin ratio (G:I) along with homeostasis model assessment (HOMA) was calculated from FG and FI level, using the formula,

$$
\mathrm{HOMA}=\frac{F G(m g \%) \times F I(m c u / m l)}{405} .
$$

Electrochemiluminescence immunoassay, Roche Lot. No. 181371-01 (Roche Diagnostics Gmbh, Mannheim, Germany) was used to measure serum TT (in $\mathrm{ng} / \mathrm{ml}$ ). SHBG level was measured $(\mathrm{nmol} / \mathrm{l})$ on the second or third day of progesterone induced withdrawal bleeding. FAI was measured using the formula,

$\mathrm{FAI}=\frac{T T \times 100 \times 3.47}{S H B G}$.

Trans-abdominal ultrasound was performed to study the morphology and volume of the ovary. Ovarian volume measurements were made as per standard protocol by measuring three perpendicular dimensions (volume for a prolate ellipsoid $=0.5 \times$ length $\times$ width $\times$ thickness of ovary). The presence of total number of follicles in each ovary was calculated both in cross-sections (longitudinal and anteroposterior) of the ovaries. Secondary causes of androgen excess like hypothyroidism, Cushing's syndrome, 21hydroxylase deficiency, androgen-secreting tumors and hyperprolactinemia were eliminated by suitable clinical and/or biochemical tests.

\section{Statistical analysis}

Presuming a confidence limit (CL) of $95 \%$, the calculated confidence interval (CI) was $10 \%$. All variables were evaluated for normality pre-test (using KS) for the comparison of all clinical and biochemical parameters detected. The continuous variables were compared with $\mathrm{t}$ test and Chi square test with Yates correction as and when needed. Logistic regression was done, (pseudo $\left.\mathrm{R}^{2}=0.3447\right)$, (Prob $\left.>\mathrm{Chi}^{2}=0.0000\right)$ predicting a good fit model $(\mathrm{p}<0.05)$ was done to assess the degree of causality.

\section{RESULTS}

In the present study, significant differences were found in the parameters of BMI ( $\mathrm{p}=0.0001)$; $\mathrm{AC}(\mathrm{p}=0.0001)$; WHR $(\mathrm{p}=0.0001) ; \operatorname{SBP}(\mathrm{p}=0.03) ; \operatorname{DBP}(\mathrm{p}=0.01) ; \mathrm{TT}(\mathrm{p}=0.002)$; FAI $(\mathrm{p}=0.002)$ between the two groups (Table 1 and 2$)$. 
Table 1: Clinical parameters in the two groups of AN present and absent in adolescent girls (Data are mean (standard deviation) or number \%).

\begin{tabular}{|llll|}
\hline Variables & AN absent $(\mathbf{n}=\mathbf{1 9})$ & AN present $(\mathbf{n}=\mathbf{4 0})$ & P value \\
\hline BMI $\left(\mathbf{k g} / \mathbf{m}^{\mathbf{2}}\right)$ & $\mathrm{N}(\%)$ & $\mathrm{N}(\%)$ & 0.0001 \\
\hline AC $(\mathbf{c m})$ & $22.8(2.4)$ & $28.1(4.4)$ & 0.0001 \\
\hline WHR $(\mathbf{c m})$ & $72.3(5.3)$ & $85.6(8.8)$ & 0.0001 \\
\hline SBP $(\mathbf{m m}$ of $\mathbf{H g})$ & $0.76(0.03)$ & $0.84(0.05)$ & 0.03 \\
\hline DBP $(\mathbf{m m}$ of $\mathbf{H g})$ & $117.7(11.03)$ & $125.0(14.5)$ & 0.01 \\
\hline
\end{tabular}

BMI, body mass index; AC, abdominal circumference; WHR, waist-hip ratio; SBP, systolic blood pressure; DBP, diastolic blood pressure.

Table 2: Hormonal and metabolic parameters of AN present and absent in adolescent girls.

\begin{tabular}{|llll|}
\hline Variables & $\begin{array}{l}\text { AN absent } \\
(\mathbf{n}=1 \mathbf{9})\end{array}$ & $\begin{array}{l}\text { AN present } \\
(\mathbf{n}=\mathbf{4 0})\end{array}$ & P value \\
\hline TT $(\mathbf{n g} / \mathbf{m l})$ & $0.39(0.13)$ & $0.62(0.43)$ & 0.002 \\
\hline SHBG $(\mathbf{n m o l} / \mathbf{l})$ & $35.8(28.1)$ & $31.8(25.4)$ & 0.60 \\
\hline FAI & $4.91(2.2)$ & $10.1(8.2)$ & 0.002 \\
\hline Number of cases with PPI $\geq \mathbf{1 5 0} \mathbf{~} \mathbf{~ c u / m l ~} \mathbf{( \% )}$ & $3(15.8)$ & $15(37.5)$ & 0.09 \\
\hline Number of cases with PPG $\geq \mathbf{1 4 0} \mathbf{~} \mathbf{m g} \mathbf{m l}(\mathbf{\%})$ & $4(21.0)$ & $8(20.0)$ & 0.92 \\
\hline
\end{tabular}

TT, total testosterone; SHBG, sex hormone binding globulin; FAI, free adrogen index; PPI, post prandial insulin; PPG, post prandial glucose.

Table 3: Demographic characteristics of patients received for study of AN.

\begin{tabular}{|c|c|c|c|c|c|}
\hline \multirow[b]{2}{*}{ S. No. } & \multirow[b]{2}{*}{ Demographic characteristics } & \multicolumn{2}{|l|}{ Frequency } & \multicolumn{2}{|c|}{ Percentage (\%) } \\
\hline & & $\begin{array}{l}\text { AN absent } \\
(\mathrm{n}=19)\end{array}$ & $\begin{array}{l}\text { AN present } \\
(n=40)\end{array}$ & $\begin{array}{l}\text { AN absent } \\
(n=19)\end{array}$ & $\begin{array}{l}\text { AN present } \\
(\mathrm{n}=40)\end{array}$ \\
\hline \multirow{7}{*}{1.} & Age of study patients & & & & \\
\hline & 14 & 2 & 5 & 11 & 13 \\
\hline & 15 & 2 & 2 & 11 & 5 \\
\hline & 16 & 4 & 9 & 21 & 23 \\
\hline & 17 & 3 & 12 & 16 & 30 \\
\hline & 18 & 4 & 7 & 21 & 18 \\
\hline & 19 & 4 & 7 & 21 & 13 \\
\hline \multirow{4}{*}{2.} & Socio-economic status & & & & \\
\hline & Low income & 4 & 11 & 21 & 28 \\
\hline & Medium income & 7 & 14 & 37 & 35 \\
\hline & High income & 8 & 15 & 42 & 38 \\
\hline \multirow{3}{*}{3.} & Habitat & & & & \\
\hline & Rural & 14 & 7 & 74 & 18 \\
\hline & Urban & 5 & 35 & 26 & 88 \\
\hline \multirow{3}{*}{4.} & Family history of DM in first $d$ & lative & & & \\
\hline & PCOS with AN & 12 & 29 & 63 & 73 \\
\hline & PCOS without AN & 7 & 11 & 37 & 28 \\
\hline
\end{tabular}

There was no significant difference in the prevalence rate of abnormal glucose tolerance $(\mathrm{p}=0.92)$ and insulin resistance between the two groups $(p=0.09)$. Logistic regression modelling with $\mathrm{AN}$ as the response variable of interest and BMI, AC, SBP, DBP, testosterone level, PP insulin and PP glucose levels as its predictors yields BMI, testosterone, PP sugar, PP insulin, SHBG as the statistically main determinants. The model signified positive impacts of BMI, testosterone level and negative influence of PP sugar, PP insulin level and SHBG levels on AN. The overall model was statistically significant with a $\mathrm{p}$ value of less than 0.000 . This analysis was also evidence for a strong relation between BMI and AN.

Demographic data revealed that both higher $(39 \%)$ and middle $(36 \%)$ income group had higher frequency of AN 
as compared to low (25\%) income group (Table 3). Also urban (64\%) adolescents showed higher frequency of ANPCOS association as compared to rural population. Similarly, a family history of DM was observed in the first degree relatives was present in $69 \%$ adolescent patients with AN and in $31 \%$ of the patients without AN.

\section{DISCUSSION}

PCOS is one of the most common gynecological endocrinal disorders of today's time affecting about 4 to $8 \%$ reproductive-aged women across the world and have been suspected to be associated with AN. ${ }^{14,15}$ However adequate studies to strongly showed such correlation have not been performed or discussed. In the present study, $68 \%$ of adolescent girls with PCOS were observed to have AN in contrast to $33 \%$ reported in adult patients with PCOS by Khan et al. ${ }^{14}$

As the sedentary lifestyle was rising, obesity in adolescence was increasingly becoming an increasing health priority as it often tracked into adulthood, resulting in enormous medical and social problems. ${ }^{3}$ Our studies showed significantly higher mean BMI $\left(28.1 \mathrm{~kg} / \mathrm{m}^{2}\right)$ in AN present group in comparison to $\mathrm{AN}$ absent group (22.8 $\mathrm{kg} / \mathrm{m}^{2}$ ). Other studies also showed that obese patients with more than $30 \mathrm{~kg} / \mathrm{m}^{2}$ BMI were more prone to develop AN. ${ }^{16}$ The study further showed that among obese PCOS patients, $58 \%$ with high BMI value (of more than 30 $\mathrm{kg} / \mathrm{m}^{2}$ ) had AN as compared to $19 \%$ patients who did not have AN. ${ }^{16}$ Similar to our study, strong correlation between $\mathrm{AN}$ and obesity had recently been reported in children as well. ${ }^{17}$ Similar to our study, low SHBG level was observed to be associated with $\mathrm{AN}$ by other researchers as well. ${ }^{18}$ WHR which indicated central obesity was high in both AN absent and present groups. However it was significantly higher in AN positive group. Similar observations in adult females were observed by other researchers as well. ${ }^{16}$ A detailed study comparing obese and nonobese PCOS showed that the percentage of AN in obese PCOS is significantly higher (91\%) as compared to nonobese PCOS (36\%). ${ }^{19}$ Obesity had been shown as an independent predictor of conversion to impaired glucose tolerance or type $2 \mathrm{DM}$ in patients with PCOS. ${ }^{19}$

Lifestyle enhancements and work patten in recent times have resulted in less physical activities, increased consumption of sugary and fatty food and alcohol in adolescents resulting in increased obesity and associated disorders. ${ }^{20}$ Weight loss, lifestyle modifications in combination with oral contraceptive pill and insulin santitizers like metformin have been considered as firstline treatment. ${ }^{21}$

Unlike our observation (69\%) Shivaprakash et al observed a family history of DM in $100 \%$ of the first degree relatives of all patients. ${ }^{16}$ AN in adolescent girl with PCOS was another clinical marker of obesity. This study showed that presence of AN was not an indicator of underlying insulin resistance or glucose intolerance.

\section{Limitations}

The major limitation of the study was that the data included in the manuscript encompasses on one selected private hospital in Eastern India which attracted more middle and higher income patients from urban setting. Thus, the demographic data may be biased. More similar studies under different demographic settings needed to be done taking into account possible variabilities.

\section{CONCLUSION}

In conclusion, this study reveals that the presence of $\mathrm{AN}$ in adolescent girls with PCOS, is an important indicator of central obesity and consequent metabolic disorders. In a low resource clinical setting, presence of $\mathrm{AN}$ in patients, should direct the clinician to suspect underlying metabolic and hormonal problems in PCOS so that we can detect early and adapt suitable precautionary steps for future metabolic complications in adolescents. This study shows that presence of $\mathrm{AN}$ is not an indicator of underlying insulin resistance or glucose intolerance. Prospective follow up studies are needed to detect how many of them ultimately develop insulin resistance or diabetes in future. Detection of AN may thus be taken to encourage lifestyle changes in the patients.

\section{ACKNOWLEDGMENTS}

The authors wish to thank S. M. Bhattacharjee for helping out with the statistical analysis and inferences.

\section{Funding: No funding sources \\ Conflict of interest: None declared}

Ethical approval: The study was approved by the Institutional Ethics Committee

\section{REFERENCES}

1. Phiske MM. An approach to acanthosis nigricans. Indian Dermatol Online J. 2014;5(3):239-49.

2. Sudevan R, Kumar SV, Sunny C. Prevalence of acanthosis nigricans and its association with physical activity in adolescents-school-based analytical crosssectional study from Kochi, Kerala. J Fam Med Prim Care. 2021;10(11):4218.

3. Ng HY. Acanthosis nigricans in obese adolescents: prevalence, impact, and management challenges. Adolesc Health Med Ther. 2017;8:1.

4. Majumdar I, Espino B, Bianco K, Epstein J, Mamilly L, Harmon CM. Multi-disciplinary weight management compared to routine care in youth with obesity: what else should be monitored? Endocrine. 2019;65(2):263-9.

5. Sadeghian G, Ziaie H, Amini M, Nilfroushzadeh M. Evaluation of insulin resistance in obese women with 
and without acanthosis nigricans. J Dermatol. 2009;36(4):209-12.

6. Wongwananuruk $\mathrm{T}$, Rattanachaiyanont $\mathrm{M}$, Indhavivadhana S. Prevalence and clinical predictors of insulin resistance in reproductive-aged thai women with polycystic ovary syndrome. Int $\mathrm{J}$ Endocrinol. 2012;2012:529184.

7. Rendon MI, Cruz PD, Sontheimer RD, Bergstresser PR. Acanthosis nigricans: a cutaneous marker of tissue resistance to insulin. J Am Acad Dermatol. 1989;21:461-9.

8. Bougrini Y, Belghol R, Elkhachine Y, Naji-Amrani $\mathrm{H}$, Ouarssani A. Acanthosis nigricans over the face revealing primitive bronchial adenocarcinoma: About a case. Pan Afr Med J. 2021;39.

9. Al-Saeed WY, Al-Dawood KM, Bukhari IA, Bahnassy A. Dermatoses in obese female schoolchildren in the Al-khobar area, eastern saudi arabia. J Family Community Med. 2006;13(2):65-9.

10. Kahn CR, Flier JS, Bar RS. The syndromes of insulin resistance and acanthosis nigricans. Insulin-receptor disorders in man. N Engl J Med. 1976;294(14):73945.

11. Hermanns-Lê T, Scheen A, Piérard GE. Acanthosis nigricans associated with insulin resistance: Pathophysiology and management. Am J Clin Dermatol. 2004;5(3):199-203.

12. Esperanza LE, Fenske NA. Hyperandrogenism, insulin resistance, and acanthosis nigricans (HAIRAN) syndrome: spontaneous remission in a 15-yearold girl. J Am Acad Dermatol. 1996;34(5):892-7.

13. Jones LH, Ficca M. Is acanthosis nigricans a reliable indicator for risk of type 2 diabetes? J Sch N. 2007;23(5):247-51.

14. Khan B, Basu R. A study on correlation between acanthosis nigricans and polycystic ovarian syndrome
(PCOS) in Indian adult women population. IOSR J Dent Med Sci. 2016;15(7):13-6.

15. Ehrmann DA. Polycystic ovary syndrome. N Engl J Med. 2005;352(12):1223-36.

16. Shivaprakash G, Basu A, Kamath A. Acanthosis Nigricansin PCOS patients and its relation with type 2 diabetes mellitus and body mass at a tertiary care hospital in Southern India. J Clin Diagn Res. 2013;7(2):317.

17. Lopez-Alvarenga JC, Chittoor G, Paul SFD. Acanthosis nigricans as a composite marker of cardiometabolic risk and its complex association with obesity and insulin resistance in Mexican American children. PLoS One. 2020;15(10):0240467.

18. Lewy V, Danadian K, Arslanian S. Sex hormone binding globulin (SHBG) and insulin-like growth factor binding protein-1 (IGF-BP1) in children with acanthosis nigricans: a marker for insulin resistance and/or hyperinsulinemia? Pediatr Res. 1998;43(4):80.

19. Silfen ME, Denburg MR, Manibo AM. Early endocrine, metabolic, and sonographic characteristics of polycystic ovary syndrome (PCOS): comparison between nonobese and obese adolescents. J Clin Endocrinol Metab. 2003;88(10):4682-8.

20. Kansra AR, Lakkunarajah S, Jay MS. Childhood and adolescent obesity: a review. Front Pediatr. 2021;8:866.

21. O'Brien B, Dahiya R, Kimble R. Hyperandrogenism, insulin resistance and acanthosis nigricans (HAIR-AN syndrome): an extreme subphenotype of polycystic ovary syndrome. BMJ Case Rep. 2020;13(4):231749.

Cite this article as: Khan B, Basu R. Acanthosis nigricans in adolescents with polycystic ovary syndrome. Int J Reprod Contracept Obstet Gynecol 2022;11:765-9. 